\title{
MEDIDAS DE PREVENÇÃO E CONTROLE DAS INFECÇÕES HOSPITALARES EM UNIDADE DE TERAPIA INTENSIVA
}

\author{
Patrícia Alves PAIVA ${ }^{1}$ \\ Priscila Karolline Rodrigues CRUZ ${ }^{2}$ \\ Fabrícia Ramos MAGALHÃES ${ }^{3}$ \\ Ricardo Soares de OLIVEIRA ${ }^{4}$ \\ Patrick Leonardo Nogueira da SILVA \\ Rogério Gonçalves da ROCHA ${ }^{6}$ \\ Wilson AGUIAR FILHO ${ }^{7}$
}

\begin{abstract}
${ }^{1}$ Enfermeira. Mestranda em Ciências da Saúde. Universidade Estadual de Montes Claros/UNIMONTES. Montes Claros (MG), Brasil. E-mail: patriciaalvespaiva@gmail.com

${ }^{2}$ Enfermeira. Universidade Estadual de Montes Claros/UNIMONTES. Montes Claros (MG), Brasil. E-mail: karolline_rcruz@yahoo.com.br

${ }^{3}$ Enfermeira. Especialista em Administração Hospitalar e Terapia Intensiva. Hospital Santa Casa de Montes Claros/HSC-MOC. Montes Claros (MG), Brasil. E-mail: fabriciaenfermagem@ hotmail.com

${ }^{4}$ Enfermeiro. Especialista em Saúde Pública, Docência do Ensino Superior e Enfermagem em Cardiologia. Secretaria Municipal de Saúde de Montes Claros/SMS-MOC. Montes Claros (MG), Brasil. E-mail: rickenfermeiromoc@yahoo.com.br

${ }^{5}$ Enfermeiro. Especialista em Saúde da Família e Didática e Metodologia do Ensino Superior. Pós-Graduando em Enfermagem do Trabalho pela Faculdade de Guanambi/FG. Guanambi (BA), Brasil. E-mail: nogueira_patrick@yahoo.com.br

${ }^{6}$ Enfermeiro. Universidade Estadual de Montes Claros/UNIMONTES. Montes Claros (MG), Brasil. E-mail: rogeriorocha81@yahoo.com.br

${ }^{7}$ Enfermeiro. Mestre em Saúde Pública. Escola Nacional de Saúde Pública Sérgio Arouca/ENSP/RJ/FIOCRUZ. Rio de Janeiro (RJ), Brasil. E-mail: aguiar.wilson@gmail.com
\end{abstract}

\section{Recebido em: 11/06/2015 - Aprovado em: 22/11/2015 - Disponibilizado em: 18/12//2015}

\begin{abstract}
RESUMO
Objetivo: identificar a importância das medidas de prevenção e controle das infecções hospitalares na Unidade de Terapia Intensiva. Método: trata-se de um estudo descritivo com suporte na revisão da literatura na qual foi realizado o levantamento das informações por meio de artigos científicos disponíveis nas seguintes bases de dados: Scielo, Lilacs e BVS, bem como em livros e revistas de saúde. Os artigos foram selecionados e analisados. Em seguida foi iniciada a discussão entre os autores. Foram selecionados 20 artigos nas bases de dados e em revistas, porém apenas 10 foram utilizados, pois os mesmos se aproximaram do tema proposto. Resultados: foram divididos em quatro categorias para a discussão, sendo as mesmas: "Unidade de Terapia Intensiva"; "Infecções hospitalares em UTI"; "Medidas de prevenção e controle das infecções hospitalares em UTI"; e "Comissões de controle de IH / Serviços de controle de IH". Conclusão: as medidas de prevenção às infecções hospitalares são constituídas pelas precauções padrão, sendo a higienização das mãos a medida isolada mais eficaz na prevenção de tal agravo; e as medidas de precauções por transmissão. A Comissão e o Serviço de Controle de IH devem estabelecer e supervisionar normas e rotinas técnicas operacionais visando reduzir e minimizar a disseminação de agentes infecciosos.
\end{abstract}

Palavras-chave: Unidade de Terapia Intensiva; Infecção Hospitalar; Prevenção Terciária.

\section{MEASURES OF PREVENTION AND CONTROL OF HOSPITAL INFECTIONS IN THE INTENSIVE CARE UNIT}

\begin{abstract}
Objective: to identify the importance of prevention and control of hospital infections in the intensive care unit. Method: this is a descriptive study with support in the literature review in which it was carried out the survey of information through scientific articles available in the following databases: Scielo, Lilacs and VHL, as well as in books and health magazines. The articles were selected and analyzed. Then began the discussion between the authors. We selected 20 articles in the databases and in magazines, but only 10 were used, as they approached the proposed theme. Results: were divided into four categories for discussion, and the same: "Intensive Care Unit"; "Nosocomial infections in ICU"; "Measures for the prevention and control of hospital infections in ICU"; and "IH control commissions / IH control services". Conclusion: prevention of hospital infections comprise the standard precautions, hand hygiene is the most effective single measure to prevent such injury; and measures of precaution transmission. The Commission and
\end{abstract}


the IH Control Service should establish and oversee operational rules and technical routines to reduce and minimize the spread of infectious agents.

Keywords: Intensive Care Units; Cross Infection; Tertiary Prevention.

\section{INTRODUÇÃO}

A Unidade de Terapia Intensiva (UTI) é o setor que concentra os principais recursos humanos e materias fundamentais para a manutenção das funções vitais dos pacientes. Ela é composta por equipes especializadas de médicos, enfermeiros e outras categorias profissionais de saúde. Leite e Vila (2005) afirmam que, os serviços de Terapia Intensiva ficam situados em áreas hospitalares destinadas ao atendimento de pacientes críticos que necessitem de cuidados complexos e especializados. Esses serviços têm como objetivos: Concentrar recursos humanos e materiais para o atendimento de pacientes graves que exigem assistência permanente, além da utilização de recursos tecnológicos apropriados para a observação e monitorização contínua das condições vitais do paciente e para a intervenção em situações de descompensações.

Para Pereira et al. (2000), a tecnologia aplicada à assistência hospitalar em Unidade de Terapia Intensiva viabiliza o prolongamento da sobrevida do enfermo em situações muito adversas. Este fenômeno altamente positivo por um lado, mas por outro, é um dos fatores determinantes do crescente risco de Infecção Hospitalar (IH) em pacientes graves. Conforme MussiPinhata e Nascimento (2001), a tendência a infecção em paciente internados em UTI é devido à combinação de vários fatores de risco em conseqüência da redução das defesas imunes e do sistema de suporte de vida, que promove ruptura de suas barreiras de defesa.

Turrini (2000) também afirma que a revolução tecnológica na assistência à saúde observada neste final de século XX trouxe muitas soluções aos agravos e possibilitou a sobrevivência de muitos pacientes graves, porém outros desafios se estabeleceram: todos os procedimentos invasivos que passaram a ser realizados levaram a indesejáveis complicações, dentre elas, as infecções hospitalares.

Turrini (2000, p. 54), ainda enfatiza que:

Os materiais invasivos utilizados são corpos estranhos colocados temporariamente ou semi-permanente no tecido do paciente com finalidade terapêutica ou diagnóstica. Estes danificam ou invadem as barreiras epiteliais e mucosas, permitindo o acesso de microrganismos diretamente na corrente sangüínea e nos tecidos. Durante o uso, facilitam o crescimento de microrganismos e agem como reservatórios de onde as bactérias podem ser transferidas para outro paciente.

E a ocorrência das infecções hospitalares varia com as características do doente, consideradas como determinantes na 
suscetibilidade às infecções. Propicia também para este fato as características do hospital, os serviços oferecidos, o tipo de paciente atendido, ou seja, a gravidade e complexidade dos pacientes, e o sistema de vigilância epidemiológica e o serviço de controle de infecções hospitalares adotados pela instituição (TURRINI, 2000). Na maioria das vezes, pouco pode se fazer em relação ao agente infeccioso ou a um hospedeiro susceptível, mas podemos interromper a cadeia de transmissão e prevenir as infecções, baseado nas medidas de controle das IH (GONÇALVES; FONSECA, 2004).

Sendo assim, este estudo objetivou identificar através da literatura a importância das medidas de prevenção e controle das infecções hospitalares na Unidade de Terapia Intensiva (UTI).

\section{MATERIAIS E MÉTODOS}

Para o desenvolvimento deste estudo e alcance dos objetivos desejados optou-se por um estudo descritivo do tipo revisão da literatura que, segundo Traldi e Dias (2011, p. 34), a da revisão da literatura busca mostrar a evolução do conhecimento sobre um determinado tema, aponta falhas e os acertos dos diversos estudos com a finalidade de encontrar pontos convergentes e divergentes, destacando o que é importante para o objetivo da pesquisa em questão. Foi realizado o levantamento das informações por meios de artigos científicos disponíveis nas bases de dados: SCIELO (Scientific Electronic Library Online), LILACS (Literatura LatinoAmericana de Ciências da Saúde), BVS (Biblioteca Virtual em Saúde), utilizando os descritores do assunto: Unidade de Terapia Intensiva, Infecção Hospitalar, Prevenção. Foram selecionados 20 artigos, nas bases de dados e em revistas porém apenas 10 foram utilizados, pois os mesmos se aproximaram do tema proposto. Também foram realizadas consultas literárias em livros. Após a seleção todos os textos e artigos foram lidos e analisados. Em seguida foi iniciada a discussão e entre os autores.

\section{RESULTADOS E DISCUSSÃO}

\section{Unidade de Terapia Intensiva}

Até a década passada, existiam poucas UTI, no Brasil, situadas apenas nas capitais dos estados e nas cidades onde ficavam as faculdades de medicina. Atualmente, grande número dos municípios com mais de 150 mil habitantes já possuem esses importantes serviços de saúde (TELAROLLI JUNIOR, 1997). As primeiras UTI surgiram a partir da necessidade de atendimento do paciente cujo estado grave exija assistência e observação continua de médicos e da equipe de enfermagem. Esta preocupação começou com Florence Nightingale, durante a Guerra da Criméia no século XIX, que procurou 
selecionar soldados atingidos mais graves, acomodando-os de forma a favorecer $\mathrm{o}$ cuidado imediato (LINO; SILVA, 2011).

Vila e Rossi (2001) também afirmam que as UTI surgiram a partir da necessidade de aperfeiçoamento e concentração de recursos materias e humanos para $\mathrm{o}$ atendimento a paciente graves, em estado critico, mas tido ainda como recuperáveis, e da necessidade de observação constante, centralizando os pacientes em um núcleo especializados. Atualmente no Brasil existem inúmeras UTI, algumas mais sofisticadas como as dos grandes centros e outros com menos recursos, porém todas possuem os requisitos básicos para o atendimento de pacientes críticos que necessitam de assistência 24 horas por dia.

Caetano et al. (2007), relata:

A UTI é tida como um local onde se
presta assistência qualificada
especializada, independentemente dos
mecanismos tecnológicos utilizados
serem cada vez mais avançados,
capazes de tornar mais eficiente o
cuidado prestado ao paciente em
estado critico, esse setor é constituído
de um conjunto de elementos
funcionalmente agrupados, destinado
ao atendimento de pacientes graves
ou de risco que exijam a assistência
médica e de enfermagem
ininterruptas, além de equipamentos e
recursos humanos especializados.

\section{Infecções Hospitalares em UTI}

O avanço da ciência, nos últimos anos, proporcionou a sobrevida das pessoas acometidas por doenças, consideradas anteriormente, como incompatíveis com a vida. No entanto, junto com essa melhoria, surgiram outros problemas, dentre eles, o aumento das infecções hospitalares (BRASIL, 2005). As infecções hospitalares constituem um sério problema de saúde desde a criação dos primeiros hospitais, quando ainda não se dispunha do conhecimento microbiológico, bem como do princípio da transmissão das doenças. A história relata sobre o médico húngaro, Ignaz Semmelweis, que em 1847, pôde perceber em sua pratica assistência a eficácia da vigilância e da adoção de medidas profiláticas, como a lavagem das mãos com solução clorada, no controle da infecção, obtendo resultados notáveis através da redução da taxa de morbimortalidade de puérperas (MARTINS, 2001).

A definição de IH segundo o Centers for Disease Control (1996) - Atlanta - EUA, refere-se a todas as infecções contraídas durante a hospitalização e que se manifeste no período da internação ou posteriormente à alta (BRASIL, 2008). Pérez e Santos (2005), definem IH, como doenças que envolvem microrganismos (bactérias, fungos, vírus, etc.), inicialmente ocorre à penetração do agente infeccioso no corpo do doente e há multiplicação dos microorganismos, com 
conseqüente apresentação de sinais e sintomas. As infecções podem acometer diversas áreas do corpo, ou disseminar na corrente sanguínea.

Para Basso e Abreu (2004), a IH é conceituada como aquela infecção adquirida após a admissão do paciente e que se manifeste durante a internação ou após a alta, quando puder ser relacionada com hospitalização ou aos procedimentos hospitalares. Quando for desconhecido o período de incubação do microorganismo e não houver evidência clinica e/ou dado laboratorial de infecção no momento da admissão, também se considera IH toda manifestação clinica de infecção que se apresentar a partir de 72 horas após a admissão.

Pereira et al. (2000), afirmam que:

$$
\begin{aligned}
& \mathrm{Na} \text { UTI concentram-se pacientes } \\
& \text { clínicos ou cirúrgicos mais graves, } \\
& \text { necessitando de monitorização e } \\
& \text { suporte contínuos de suas funções } \\
& \text { vitais. Este tipo de clientela apresenta } \\
& \text { doenças ou condições clínicas } \\
& \text { predisponentes a infecções. Muitos } \\
& \text { deles já se encontram infectados ao } \\
& \text { serem admitidos na unidade e, a } \\
& \text { absoluta maioria, é submetida a } \\
& \text { procedimentos invasivos ou } \\
& \text { imunossupressivos com finalidades } \\
& \text { diagnostica e terapêutica. }
\end{aligned}
$$

Para o paciente criticamente doente, o uso de tecnologia invasiva muitas das vezes é primordial. No entanto, para que o paciente se beneficie desses avanços tecnológicos, é essencialmente importante que os profissionais de saúde que assistem estejam muito bem capacitados e conscientes da extensão e gravidade da enfermidade do doente submetido à terapia intensiva (MUSSIPINHATA, 2001). A associação de patologias e fatores iatrogênicos faz com que o doente seja mais susceptível à aquisição de infecções hospitalares. A resposta imunológica do paciente em Terapia Intensiva frente ao processo infeccioso é deficiente. Os seus mecanismos de defesa estão debilitados tanto pela doença motivadora da hospitalização quanto pelas intervenções necessárias para o diagnóstico e tratamento (PEREIRA et al., 2000).

E para Fernandes et al. (2000), a grande maioria das IH ocorrem por um desequilíbrio da relação existente entre a microbiota humana normal e os mecanismos de defesa do paciente. Isto poder ser devido a patologia de base do paciente, procedimentos invasivos e alterações microbiana, geralmente induzida pelo o uso dos antibióticos. O paciente poderá ser infectado após sua chegada a UTI por várias vias, incluído a equpipe de saúde, o contato direto e indireto, as gotículas e os materiais e equipamentos infectados.

A IH acontecerá através do contanto direto (transmissão física direta de microorganismo de uma pessoa colonizada ou infectada para o hospedeiro, por meio das mãos ou secreções), ou pelo contato indireto 
(transmissão por objetos inanimados) e também através da transmissão área por gotículas. (FERNANDES et al., 2000). Segundo estatísticas do Governo Federal atualmente $15 \%$ dos pacientes hospitalizados no país contraem algum tipo de $\mathrm{IH}$, mas existem casos em que $30 \%$ das infecções hospitalares podem ser prevenidas. Sendo a pneumonia, infecções cirúrgicas, sepses e infecções urinarias as principais infecções mais presentes. (RODRIGUES et al., 1997)

Para Machado (2001), a IH representa um problema de qualidade de assistência devido a importância incidência, letalidade, aumento no tempo de hospitalização e no aumento do consumo de medicamentos, além dos custos indiretos com aqueles representados pela cessação da produção do paciente e custos inatingíveis ou difíceis de avaliar economicamente, como distúrbios pela dor, mal-estar, isolamento, enfim, pelo aumento do sofrimento do doente.

Corroboram Chianca et al. (2002), também afirmando que as IH além de representarem importante causa de morbidade, mortaliade e significantes aumento nos custos assistenciais, torna-se imperativo a implementação de ações de prevenção controle e avaliação dessas infecções. Também se considera que a prevenção da IH no ambiente hospitalar ocorra em um nível individual, pois os pacientes com baixa imunidade encontram-se concentradas em mesmo local, sendo fontes de infecção para si mesmas, para outros pacientes e para o próprio ambiente, à medida que as transmissões de microorganismo ocorrem pelo contato físico direto por meio das mãos, pelo contato com objetos contaminados.

\section{Medidas de prevenção e controle das infecções hospitalares em UTI}

Atualmente, os usuários dos serviços hospitalares antes de receber à assistência possuem o direito de serem esclarecidos sobre as porcentagens de infecções hospitalares da instituição. Tais dados são computados e oferecidos a partir dos serviços da CCIH. Diante disso, os profissionais de saúde devem se preocupação quanto à necessidade de se respeitar e esclarecer o direito que o usuário tem de interna-se em um hospital sem risco de adquirir uma IH (SOUZA et al., 2008).

Portanto, ações e medidas de prevenção as infecções hospitalares devem ser rotinas em todas as instituições que se preocupam com a satisfação da sua clientela e que têm responsabilidade com a saúde. É no ambiente de Terapia Intensiva, é indicado que essas ações de prevenção ao controle de infecções sejam mais rigorosas, em decorrência do tipo de paciente, do ambiente e da intensidade dos procedimentos invasivos. São inúmeras as medidas de prevenção às infecções hospitalares, porém as mesmas devem ser conhecidas e praticadas por todos aqueles que irão lidar diretamente ou indiretamente ao paciente. 
Martins (2001), afirma que as medidas de prevenção as infecções a serem adotadas pelos serviços hospitalares são as de Precauções Padrão e Precações Baseadas na Transmissão. Sendo as Precações Padrão constituídas pela Lavagem das mãos; Uso correto das luvas, Máscaras, Protetor facial e de olhos, utilização de roupas de proteção, manuseio adequado dos materias perfurocortantes e cuidado com os equipamentos e demais matérias.

Mercedes (2002) relata que o objetivo das precauções padronizadas é buscar a redução da transmissão de patógenos a partir de fontes de infecção conhecidas ou desconhecidas. As precauções Padrão enfatizam condutas como, higienização das mãos, uso de luvas, máscaras, protetor facial e de olhos, roupas de proteção, manuseio cuidadoso dos instrumentos perfuro-cortantes e cuidado com materias e equipamentos.

De acordo com Souza et al., (2008), alguns médicos acreditam que a pratica dos profissionais de saúde, esta bastante relacionada com a adoção das medidas de biossegurança, o uso dos equipamentos de proteção individual (EPI) e a lavagem das mãos. A higienização das mãos, nos programas de prevenção e controle das infecções hospitalares, é uma pratica prioritária, considerando ser uma ação isoladamente mais importante para reduzir as taxas dessas infecções. Sendo que o principal objetivo do processo de higienização das mãos é o de reduzir a transmissão de microorganismo pelas mãos, prevenindo as infecções.
E a utilização das medidas e técnicas específicas para impedir a transmissão de infecções entre as pessoas não é recente, sendo encontrados em relatos do século XVII descrevendo as estratégias utilizados para conter a epidemia de peste bubônica. (BERHMAN et al., 2000). Minas Gerais (2005) enfatiza que apesar da tendência do crescimento da complexidade dos pacientes internados e, conseqüentemente, o aumento das infecções hospitalares, as medidas tradicionais de prevenção permanecem como as fundamentais estratégias de controle às infecções. Essas ações devem ser instituídas através do conhecimento dos dados gerados, pelo processo de vigilância epidemiológica, adequando-as necessidades específicas de cada serviço.

Os antimicrobianos também possuem importante papel no contexto das infecções hospitalares. Com a evolução da tecnologia, os antimicrobianos foram aperfeiçoados, técnicas de assistência foram se desenvolvendo e o tratamento das patologias assumiu um grau de alta complexidade. Por outro lado, a invasão das bactérias multirresistentes, a inserção de novas formas de microorganismo e a luta contra a resistência bacteriana surgiram nesse contexto, fragilizando o ambiente do cuidado das pessoas e desafiando as ações do cotidiano dos profissionais de saúde, no que se refere à prevenção das infecções hospitalares. (BRASIL, 2000). 


\section{Comissão de controle de IH / Serviço de controle de IH}

A IH representa uma preocupação não somente dos órgãos de saúde competente, mas como um problema de ordem social, ética e jurídica em face às implicações na vida dos pacientes e o risco que estes estão expostos. Desta forma a IH tanto no Brasil como nos outros países significa importante problema de saúde publica (LACERDA, 2003).

Devido ao fato de que as $\mathrm{IH}$ são consideradas um problema de Saúde Publica, institui a portaria 140 do Ministério da Saúde, publicada em 8 de Abril de 1987, onde ela cria Comissão Nacional de Controle das IH; a portaria 232, de 6 de abril de 1988, institui o Programa Nacional de Controle de Infecção ligadas á secretaria Nacional de Programas especiais; e também são criadas as primeiras Associações de Profissionais em Controle de IH do estado de São Paulo (APECIH-SP); Associação Mineira em Controle de IH (AMECIH-MG); Associação Brasileira de Controle de IH (ABCIH) (MARTINS, 2005).

Desde 1983 que o controle das IH é regido por ações governamentais desde 1983. Mas somente em 1997 a existência de um Programa de Controle das Infecções Hospitalares (PCIH) em todas as instituições hospitalares do país, que passou a ser obrigatória pela Lei $\mathrm{n}^{\circ}$ 9.431/97. E em 1998 foi editada a portaria, $\mathrm{n}^{\mathrm{o}}$ 2.616, que recomenda o processo de trabalho a ser realizado pelo o PCIH, devendo a instituição constitui a $\mathrm{CCIH}$, um órgão deliberativo de ações de controle e prevenção de $\mathrm{IH}$ e o Serviço de Controle de IH, denominada $\mathrm{SCIH}$, órgão executivo, que encarrega de realizar as ações deliberadas pela $\mathrm{CCIH}$ (BRASIL, 2000).

A Comissão de Controle das Infecções Hospitalares é responsável por realizar a vigilância epidemiológica de todos os pacientes, principalmente daqueles que se apresentam um risco maior para infecção. A vigilância é um processo que ocorre por meios de métodos prospectivos, como a busca ativa, sistemática e continua das infecções, bem como sua propagação (BRASIL, 2000). As instituições hospitalares através das $\mathrm{CCIH}$ e dos SCIH devem criar implantar e supervisionar normas e rotinas técnicas operacionais com objetivo de reduzir e minimizar a disseminação dos agentes infecciosos (COUTO, 2003).

Mesmo com a criação de inúmeras portarias já publicadas ainda é reduzido o número de instituições hospitalares que possuem uma comissão ou serviço de controle das infecções hospitalares funcionando de maneira efetiva. Atualmente, quase duas décadas após a portaria 196 a situação ainda é precária havendo uma concentração de bons serviços de controle de IH nos hospitais de grande porte e uma deficiência nos hospitais de periferia e nos hospitais do interior do estado (MARTINS, 2005). 
Mesmo com a legislação vigente, os dados sobre a IH são pouco divulgados devido os dados não serem consolidados por muitas instituições hospitalares, o que tem dificultado a aquisição de um diagnóstico que retrate a dimensão desse agravo (TURRINI, 2000). Mesmo com a legislação, os índices de IH são altos, $15 \% 5$ o que corresponde a $1,18 \%$ de infecção por paciente internado com IH. Além disso, consideram-se mais um agravante, o fato das instituições publicas possuir a maior taxa de prevalência de $\mathrm{IH}$, de 18,4\% (LACERDA, 2002).

Para Lacerda (2002), a problemática da IH deve se ampliam a perspectiva de sua determinação social, ou, seja as suas práticas de prevenção e controle não dependem apenas de ações focais, no âmbito restrito de um Programa de Controle das Infecções Hospitalares (PCIH). Dependem também de ações ampliadas e relacionadas às formas com que as políticas de saúde são introduzidas e distribuídas, à qualidade da assistência, a reformulação ou inovação de modelos técnicos-assistencias é a elaboração de estratégias de avaliação.

Portanto é imprescindível a vigilância epidemiológica das infecções hospitalares através da observação ativa, sistemática e contínua de sua freqüência e distribuição entre os pacientes e dos eventos e condições que afetam o risco de seu surgimento, com vistas à execução oportuna das ações de prevenção e controle. Trata-se de um processo que compreende a coleta, consolidação, análise e interpretação dos dados observados, gerando informações essenciais para planejamento, implementação de ações e avaliação de medidas de intervenção através da supervisão da assistência dos profissionais de saúde (MARTINS, 2005). É pertinente enfatizar a importância da educação continuada, pois a organização e execução de atividades educativas contribuirão para a melhoria e controle da qualidade da assistência. Pois no processo da prevenção da $\mathrm{IH}$, os recursos humanos são a base para o sucesso.

\section{CONSIDERAÇÕES FINAIS}

A partir da literatura científica foi possível conhecer historicamente o inicio dos primeiros atendimentos intensivos que surgiram desde a época da pioneira Florence Nightingale durante a Guerra da Criméia no século XIX. Pode-se constatar que o grande avanço da tecnologia voltada para a assistência hospitalar em Unidade de Terapia Intensiva tem proporcionado o prolongamento da sobrevida dos pacientes em estado grave e crítico. Mas em contrapartida surgiu também o crescente risco de $\mathrm{IH}$ nesse pacientes. Tornando esse evento um problema de problema de saúde pública no Brasil e no mundo.

A IH é compreendida como toda infecção adquirida após o ato da admissão do paciente e que se manifeste durante o período 
de hospitalização ou após a alta (e que esteja relacionada com hospitalização ou com os procedimentos). Também só será considera IH se as manifestações clínicas apresentarem a partir das 72 horas da internação. Uma vez instalada uma $\mathrm{IH}$, várias situações negativas surgiram, sendo algumas delas o, aumento da morbimortalidade, no tempo de internação, no consumo de medicamentos, e conseqüentemente no aumento dos gastos da instituição. Sem contar no prejuízo do bem estar do paciente e da família.

Portando, ações de prevenção e de controle das infecções hospitalares são requisitos indispensáveis na assistência ao paciente de Unidades de Terapia Intensiva. Essas ações devem buscar a redução da transmissão de microorganismos a partir de fontes de infecção conhecidas ou desconhecidas. Denominam ações de controle e prevenção de infecções hospitalares a utilização das medidas de Precações Padrão, constituídas pela Higienização das mãos; uso correto das luvas, máscaras, protetor facial e de olhos, utilização de roupas de proteção, manuseio adequado dos materias perfurocortantes, equipamentos e matérias. $\mathrm{E}$ as Precauções Baseadas na Transmissão, constituídas por medidas adequadas para cada tipo de transmissão. Sendo que de todas as medidas a Higienização das mãos é a mais importante, devido às mãos serem um veículo de microorganismo
Além da realização efetiva das medidas de controle de prevenção da IH, deve ser realizada a vigilância epidemiológica de todos os pacientes, principalmente daqueles que se apresentam um risco maior para infecção. A vigilância epidemiológica constitui na busca ativa, sistemática e continua das infecções. Para essa tarefa toda instituição deve contar com uma CCIH, e o SCIH, que são respectivamente comissão e serviço de controle de IH, sendo eles responsáveis pela padronização de medidas de prevenção e execução de atividades educativas com o objetivo de capacitar os profissionais de saúde que prestam a assistência.

\section{REFERÊNCIAS}

BASSO, M.; ABREU, E. S. Limpeza, desinfecção de artigos e áreas hospitalares e anti-sepsia. $2^{a}$ ed. São Paulo: Associação Paulista de Estudos e Controle de Infecção Hospitalar; 2004.

BERHMAN, R. E. et al. Tratado de pediatria. $16^{a}$ ed. Rio de Janeiro: Guanabara Koogan; 2002.

BRASIL. Ministério da Saúde. Programa de Controle de Infecção Hospitalar. Relatório 2008. Brasília: PCIH; 2008.

BRASIL. Ministério da Saúde. Agência Nacional de Vigilância Sanitária. Prevenção e controle de infecção hospitalar. Brasília: ANVISA; 2005.

BRASIL. Ministério da Saúde. Portaria no 196 de 24/07/1983. In: BRASIL. Ministério da Saúde. Manual de controle de infecção 
hospitalar. Brasília: Centro de

Documentação do Ministério da Saúde; 2000.

CDC. Centers for Disease Control and Prevention. National Center for Health Statistics. Growth Charts: United States. Hyaltsville; 1996.

CAETANO, J. A. et al. Cuidado humanizado em terapia intensiva: um estudo reflexivo.

Revista Escola Anna Nery. Rio de Janeiro, v. 11, n. 2, p. 325-330, 2007.

CHIANCA, T. C. M.; ERCOLENE, F. F.; OLIVEIRA, A. C. As comissões de infecção hospitalar e as unidades de centro cirúrgico: uma reflexão histórico-critica acerca da prática. Revista Nursing (São Paulo). São Paulo, v. 5, n. 46, p. 22-28, 2002.

COUTO, R. C. et al. Infecção hospitalar epidemiologia e controle. $3^{\mathrm{a}}$ ed. Rio de Janeiro: Medsi; 2003.

FERNANDES, A. T. et al. Conceito, cadeia epidemiológica das infecções hospitalares e avaliação custo beneficio das medidas de controle. In: FERNANDES, A. T.;

FERNANDES, M. O. V.; RIBEIRO, F. N. (org.). Infecção hospitalar e suas interfaces na área de saúde. São Paulo: Atheneu; 2000.

GONÇALVES, M. B.; FONSECA, A. S. Visão de docentes de enfermagem quanto aos cuidados básicos para a prevenção de infecção. Revista Nursing (São Paulo). São Paulo, v. 7, n. 78, p. 23-28, 2004.

LACERDA, R. A. Infecção hospitalar e sua relação com a evolução das práticas de assistência em saúde. In: LACERDA, R. A. Controle das infecções em centro cirúrgico: fatos, mitos e controvérsia. São Paulo: Atheneu; 2003.

LACERDA, R. A. Produção científica nacional sobre infecção hospitalar e a contribuição da enfermagem: ontem, hoje e perspectivas. Revista Latino-Americana de Enfermagem. Ribeirão Preto, v. 10, n. 1, p. 55-63, 2002.
LEITE, M. A; VILA, V. S. C. Dificuldades vivenciadas pela equipe multiprofissional na unidade de terapia intensiva. Revista LatinoAmericana Enfermagem. Ribeirão Preto, v. 13, n. 12, p. 145-150, 2005.

LINO, M. M.; SILVA, S. Enfermagem na unidade de terapia intensiva: a história como explicação de uma prática. Revista Nursing (São Paulo). São Paulo, v. 41, n. 4, p. 25-29, 2011.

MACHADO, G. P. M. et al. Aspectos epidemiológicos das infecções hospitalares. In: MARTINS, M. A. Manual de infecção hospitalar: epidemiologia, prevenção e controle. $2^{\mathrm{a}}$ ed. Rio de Janeiro: Medsi; 2001.

MARTINS. M. A. Aspectos históricos da infecção hospitalar. In: OLIVEIRA, A. C. Infecções hospitalares, prevenção e controle. $1^{\mathrm{a}}$ ed. Rio de Janeiro: Guanabara Koogan; 2005.

MARTINS, M. A. Manual de infecção hospitalar: epidemiologia, prevenção e controle. $2^{\mathrm{a}}$ ed. Rio de Janeiro: Medsi; 2001.

MERCEDES, A. L. Guia prático de enfermagem - Hospitalização: prevenção das infecções hospitalares. Rio de Janeiro: Mc Graw Hill; 2002.

MINAS GERAIS. Cartilhas da Secretaria de Estado da Saúde de Minas Gerais e do Hospital das Clínicas/UFMG. Belo Horizonte; 2005.

MUSSI-PINHATA, M. M.; NASCIMENTO, S. D. Infecções neonatais hospitalares. Jornal de Pediatria (Rio de Janeiro). Rio de Janeiro, v. 77, supl. 1, p. 81-96, 2001.

PEREIRA, M. S. et al. Controle de infecção hospitalar em unidade de terapia intensiva: desafios e perspectivas. Revista Eletrônica de Enfermagem. Goiânia, v. 2, n. 1, 2000.

PÉREZ, A. U. R.; SANTOS, L. S. Infección nosocomial: impacto y perspectivas. Revista 
Mexicana de Patologia Clínica. Cuba, v. 52,

n. 3, p. 168-170, 2005.

RODRIGUES, E. A. C. et al. Infecções hospitalares, prevenção e controle. São

Paulo: Sarvier; 1997.

SOUZA, A. C. S. et al. Os direitos dos usuários da saúde em casos de infecção hospitalar. Revista Brasileira de

Enfermagem. Brasília, v. 61, n. 4, p. 411417, 2008.

TELAROLLI JUNIOR, R. Mortalidade infantil: uma questão de saúde pública. $2^{\mathrm{a}}$ ed. São Paulo: Moderna; 1997.

TRALDI, M. C.; DIAS, R. Monografia Passo a Passo. Edição Especial. Campinas: Alínea; 2011.

TURRINI, R. N. T. Percepção das Enfermeiras sobre fatores de risco para a infecção hospitalar. Revista da Escola de Enfermagem da USP. São Paulo, v. 34, n. 2, p. 174-184, 2000.

VILA, V. S. C.; ROSSI, L. A. O significado cultural do cuidado humanizado em unidade de terapia intensiva: "muito falado e pouco vivido". Revista Latino-Americana de Enfermagem. Ribeirão Preto, v. 10, n. 2, p. 137-144, 2001. 